Supporting Information for Publication:

\title{
Heavily doped Semiconductor Colloidal Nanocrystals as Ultra-Broadband Switches for Near Infrared and Mid-Infrared Pulse Lasers
}

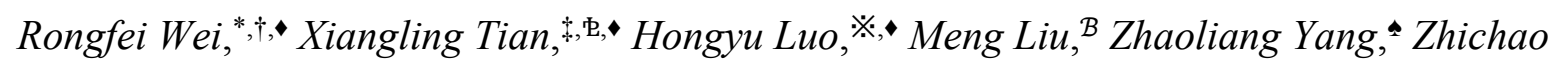
Luo ${ }^{\mathcal{B}}$ Haiming Zhu, Hai Guo,${ }^{\dagger}$ Jianfeng $L i^{*}, ※$ and Jianrong Qiu ${ }^{*, \downarrow, \mathrm{E}}$

${ }^{\dagger}$ Department of Physics, Zhejiang Normal University, Jinhua, Zhejiang, 321004, PR China

State Key Laboratory of Luminescent Materials and Devices and School of Materials Science and Engineering, South China University of Technology, Wushan Road 381, Guangzhou 510641, PR China

'Division of Physics and Applied Physics, School of Physical and Mathematical Sciences, Nanyang Technological University, 21 Nanyang Link, Singapore 637371, Singapore

※School of Optoelectronic Science and Engineering, University of Electronic Science and Technology of China, Chengdu, Sichuan, 610054, PR China

${ }^{\mathcal{B}}$ School of Information and Optoelectronic Science and Engineering, South China Normal University, No.378, West Waihuan Road, Guangzhou, 510006, PR China

^State Key Laboratory of Chemical Engineering, Center for Chemistry of High-Performance 
\& Novel Materials, Department of Chemistry, Zhejiang University, Hangzhou 310027, PR

\section{China}

${ }^{\circledR}$ State Key Laboratory of Modern Optical Instrumentation, College of Optical Science and Engineering, Zhejiang University, Hangzhou, Zhejiang 310027, PR China

Correspondence should be addressed to: R.W.(rfwei@zjnu.edu.cn); J. L.

(lijianfeng@uestc.edu.cn); J. Q.(qjr@scut.edu.cn)

$\bullet$ R. Wei, X. Tian and H. Luo contribute equally 


\section{CALCULATION OF HOLE DENSITY AND LSPR FREQUENCY}

The plasmonic resonance frequency can be deduced using the Drude-Lorentz model. ${ }^{1}$ The dipole polarizability $(\alpha)$ of heavily doped semiconductor nanocrystals can be described by the following equation:

$\alpha=3 \varepsilon_{0} v\left(\frac{\varepsilon-\varepsilon_{\mathrm{m}}}{\varepsilon+2 \varepsilon_{\mathrm{m}}}\right)$,

where, $\varepsilon_{0}$ is the free space permittivity, $\varepsilon_{\mathrm{m}}$ is the dielectric constant of the surrounding medium, $v$ is the particle volume. $\varepsilon$ is the dielectric function of heavily doped semiconductor nanocrystals and can be described as $\varepsilon(\omega)=\varepsilon_{\mathrm{r}}(\omega)+i \varepsilon_{\mathrm{i}}(\omega)$, in which, $\varepsilon_{\mathrm{r}}$ and $\varepsilon_{\mathrm{i}}$ are the real part and imaginary part of dielectric function, respectively. Because of the broad band-gap in $\mathrm{Cu}_{1.8} \mathrm{Se}(3.36 \mathrm{eV})$, the dielectric function of $\mathrm{Cu}_{1.8} \mathrm{Se}$ in near-infrared (NIR) region is determined by free carriers.

The resonant condition can be achieved under the condition of $\varepsilon_{\mathrm{r}}=-2 \varepsilon_{\mathrm{m}}$. The real part $\varepsilon_{\mathrm{r}}$ is a function of the frequency of light and can be describe as:

$\varepsilon_{\mathrm{r}}=1-\frac{\omega_{\mathrm{p}}^{2}}{\omega^{2}+\gamma^{2}}$,

in which, $\omega, \omega_{\mathrm{p}}$ and $\gamma$ are the angular frequency (in $\operatorname{rad~s}^{-1}$ ), the plasma frequency (bulk plasma oscillation) associated with the free carriers and the electron damping rate (bulk collision frequency), respectively. Hence, the LSPRs frequency, $\omega_{\mathrm{sp}}$, can be deduced as:

$$
\omega_{\mathrm{sp}}=\sqrt{\frac{\omega_{\mathrm{p}}^{2}}{1+2 \varepsilon_{\mathrm{m}}}-\gamma^{2}},
$$


The collision frequency is in numerically experimental determined width of the LSPRs. ${ }^{1}$ The calculated LSPRs frequency is about $1.1 \times 10^{15} \mathrm{~Hz}$, and the extracted LSPRs wavelength is $1713 \mathrm{~nm}$.

The carrier density can be extracted from the following relationship:

$\omega_{\mathrm{p}}=\sqrt{\frac{N_{\mathrm{h}} e^{2}}{\varepsilon_{0} m^{*}}}$,

here, $N_{\mathrm{h}}$ is the hole density in the prepared $\mathrm{Cu}_{1.8} \mathrm{Se}, e$ presents the electron charge, and $\mathrm{m}^{*}$ describes the effective mass $\left(\mathrm{m}^{*}=0.336 \mathrm{~m}_{0}\right.$, and $\mathrm{m}_{0}$ is the electron mass) in $\mathrm{Cu}_{1.8} \mathrm{Se}^{2}{ }^{2}$ The calculated hole density is about $1.56 \times 10^{20} \mathrm{~cm}^{-3}$.

2. CHARACTERIZATION

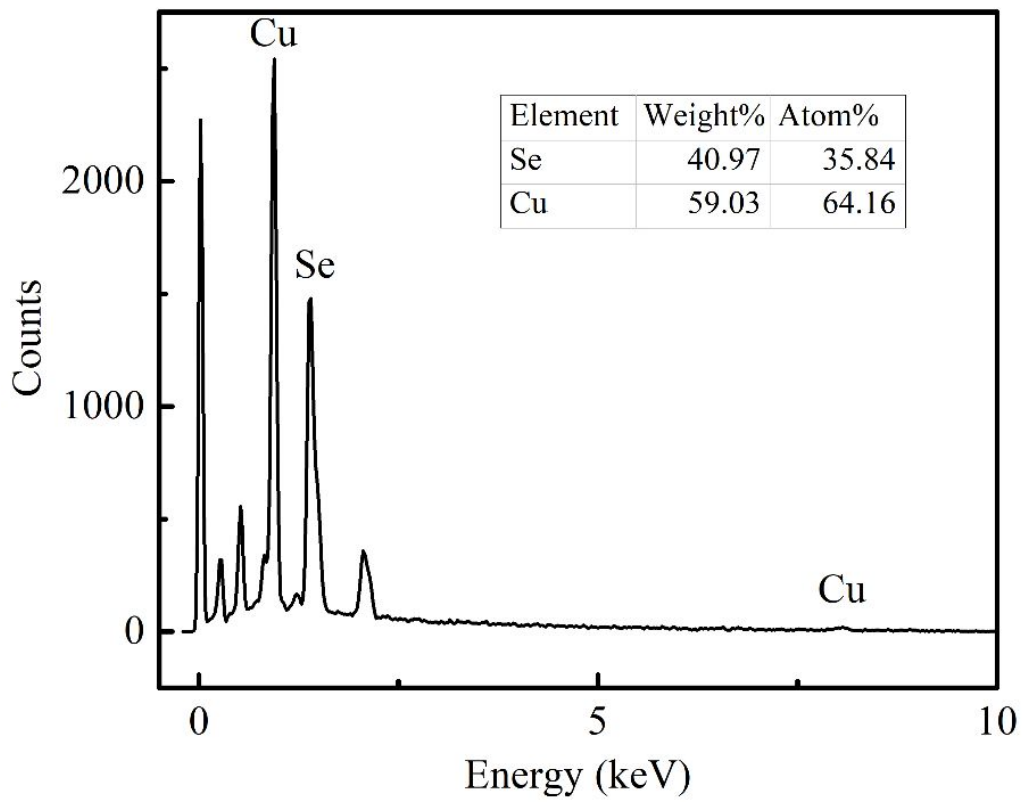

Figure S1. The result spectrum of EDX. Inset: the atom proportion of the $\mathrm{Cu}$ and $\mathrm{Se}$. 


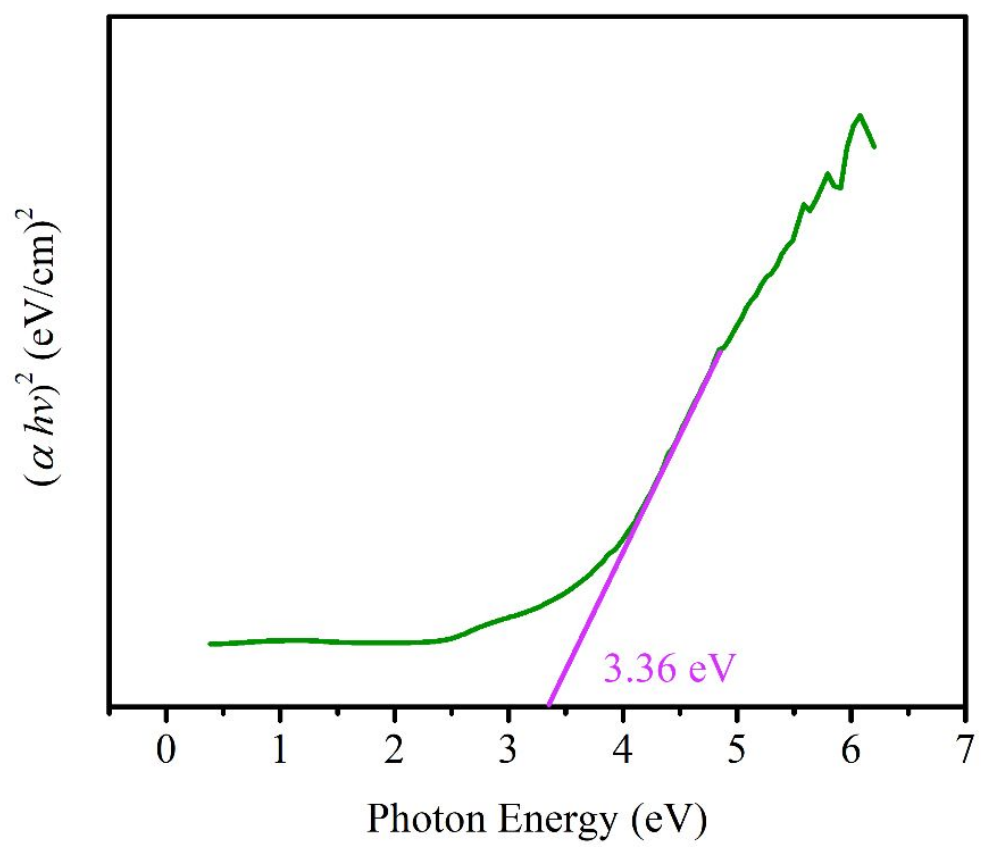

Figure S2. According to the Kubelka-Munk function versus energy of light $(h v),{ }^{3}$ the linear region in the relationship between $(\alpha h v)^{2}$ and $h v$ shows the bandgap of $\mathrm{Cu}_{1.8}$ Se nanocrystals is about $3.36 \mathrm{eV}$. Here, the absorption coefficient $(\alpha)$ is calculated by the formula of $\alpha=\ln (1 / T) / L{ }^{4} T$ and $L$ are the linear transmittance and light path-length (sample thickness).

a

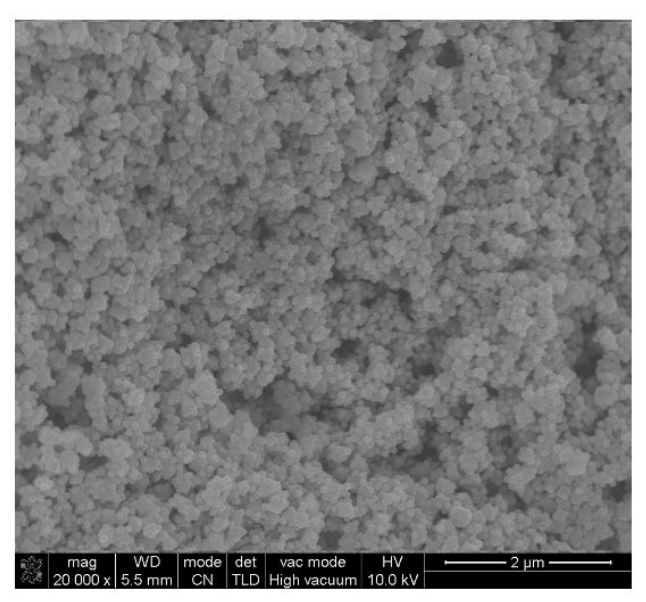

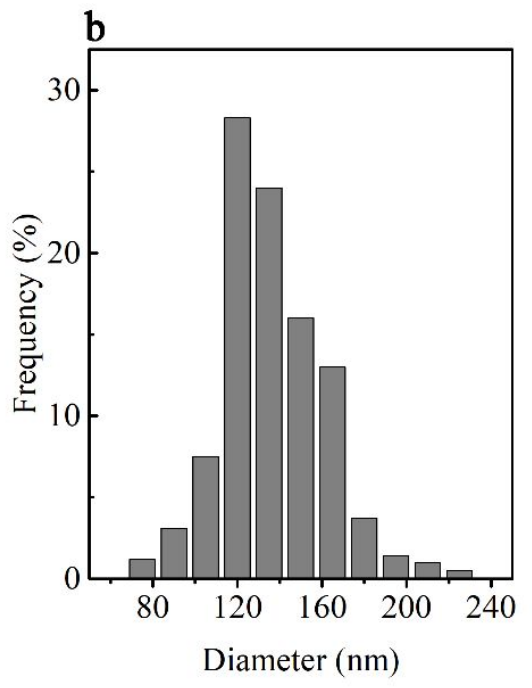

Figure S3. (a) SEM image of $\mathrm{Cu}_{1.8}$ Se nanocrystals. (b) The size distribution based on the SEM image in (a). 


\section{PULSE GENERATION}

$1550 \mathrm{~nm}$ fiber laser setup: The schematic of the pulsed fiber laser can be found in our previous work. ${ }^{5}$ In short: the gain medium is a piece of $4 \mathrm{~m}$ erbium-doped fiber (EDF) with a dispersion parameter of $-17.3 \mathrm{ps} / \mathrm{km} / \mathrm{nm}$. The other fibers were standards single mode fiber (SMF). An integrated device containing a PI-ISO, WDM and 10\% output coupler was employed to make the laser cavity more concise, and to make the pump laser couple into the cavity, ensure unidirectional light propagation and output the laser. A PC was used to adjust the polarization states of the propagation light. An optical spectrum analyzer (OSA, Yokogawa AQ6317C) and a high-speed real-time oscilloscope (Tektronix DSA-70804, 8 $\mathrm{GHz}$ ) with a photodetector (Newport 818-BB-35F, $12.5 \mathrm{GHz}$ ) were used to monitored the laser performance. In addition, an autocorrelator (FR-103XL) was employed to measure the pulse duration.

$2000 \mathrm{~nm}$ fiber laser setup: Figure S4 shows the experimental setup of passively Q-switched Tm-doped fiber (TDF) laser cavity based on $\mathrm{Cu}_{1.8} \mathrm{Se}$ NCs saturable absorber. A commercial $793 \mathrm{~nm}$ laser diode (LD) (BWT, Beijing), which has a $105 \mu \mathrm{m}$ core diameter and a numerical aperture (NA) of 0.22 was used as the pump source. The pump laser was launched into the gain fiber through a $(2+1) \times 1$ combiner (IFT, Canada). The gain fiber was a piece of $2.0 \mathrm{~m}$ double-cladding TDF (Coractive, DCF-TM-10/128) with an octagonal shaped inner cladding with a diameter of $128 \mu \mathrm{m}$ and a NA of 0.45 , and a circular core with a diameter of $10 \mu \mathrm{m}$ 
diameter and a NA of 0.22 . The cladding absorption coefficient of $\sim 4 \mathrm{~dB} / \mathrm{m}$ measured at 793 nm gave a nominally 94\% total absorption at this length. A 50:50 coupler (IFT, Canada) was spliced with the TDF to output half of the signal laser. PI-ISO was used to maintain the intracavity laser propagate unidirectionally. $\mathrm{The} \mathrm{Cu}_{1.8} \mathrm{Se} /$ poly(vinyl alcohol) (PVA) film sandwiched between two FC/PC connectors was used as the saturable absorber device. A PC was placed between the combiner and saturable absorber device to control the intra-cavity loss. When the intensity of the propagation light in the fiber waveguide is large enough for the $\mathrm{Cu}_{1.8} \mathrm{Se} / \mathrm{PVA}$ film to trigger a Q-switching operation, stable Q-switching regime with a low amplitude fluctuation of $\pm 3 \%$ was obtained. The temporal pulse train and single pulse waveforms were monitored using a InGaAs photodetector (EOT ET-5000F, USA) equipped with an $8 \mathrm{GHz}$ digital oscilloscope. The radio frequency (RF) spectrum was performed by a RF spectrum analyzer (YIAI, China, AV4033A, 30Hz-18GHz). The optical spectrum was investigated using an optical spectrum analyzer (Yokogawa AQ6375, Japan) with a resolution of $0.05 \mathrm{~nm}$. The output power was recorded using a thermal power sensor (S470C, Thorlabs, USA).

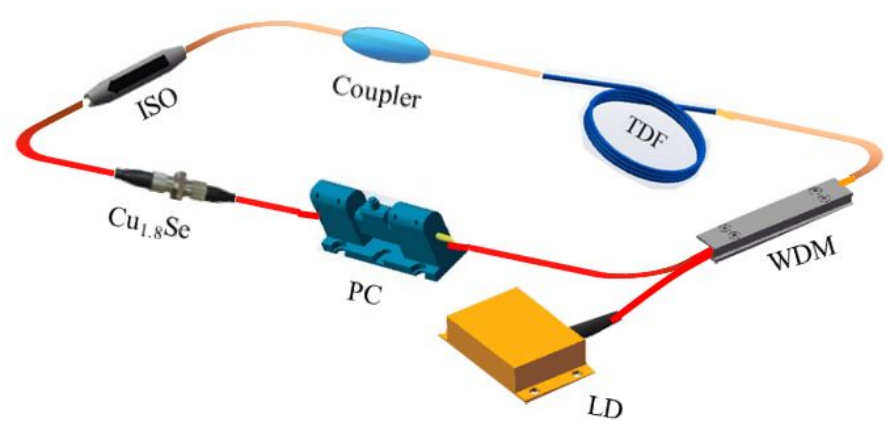

Figure S4. Schematic illustration of a passively Q-switched Tm-doped fiber laser constructing a ring cavity using $\mathrm{Cu}_{1.8} \mathrm{Se} / \mathrm{PVA}$ as saturable absorber. 
$3000 \mathrm{~nm}$ fiber laser setup: The schematic of the pulsed fiber laser can be found in our previous work. ${ }^{6}$ In short, two commercially available diode lasers (1150 nm, Eagleyard Photonics, Berlin) were adopted as pump source. A commercial double-cladding $\mathrm{Ho}^{3+} / \mathrm{Pr}^{3+}-$ codoped ZBLAN fiber with the concentration of $\mathrm{Ho}^{3+}-30000$ and $\mathrm{Pr}^{3+}-2500 \mathrm{ppm}$ (fiberlabs, Japan) was used as the gain medium, which has an octangular pump core with a diameter of $125 \mu \mathrm{m}$ and a NA of 0.5 , and a circular core with a diameter of $125 \mu \mathrm{m}$ and a NA of 0.2 . An uncoated ZnSe objective lens (focal length: $6 \mathrm{~mm}$, Innovation Photonics, LFO-5-6, 0.25 NA) was firstly used to collimate the output of the laser. After that, the output was then focused by another same $\mathrm{ZnSe}$ objective lens terminated with the $\mathrm{Cu}_{1.8} \mathrm{Se} / \mathrm{PVA}$ composite film (coated commercial gold-protected mirror). An InAs detector with a response time of 2 ns equipped with a $500 \mathrm{MHz}$ bandwidth digital oscilloscope was adopted to monitor temporal pulse trains and waveforms. A RF spectrum analyzer (AV4033A) with a scanning range of $30 \mathrm{~Hz}-18 \mathrm{GHz}$ was used to record the RF spectrum. A monochromator (Princeton instrument Acton SP2300, scanning resolution: $0.1 \mathrm{~nm}$ ) was used to identify the optical spectrum.

\section{NONLINEAR ABSORPTION (NLA) MEASUREMENT}

NLA measurement at $2000 \mathrm{~nm}$ : Figure S5 shows the schematic of arrangement used for nonlinear absorption measurement of $\mathrm{Cu}_{1.8} \mathrm{Se} / \mathrm{PVA}$ composite film at $2000 \mathrm{~nm}$. The laser source was an in-house ultrashort pulse fiber laser system including a nonlinear polarization rotation (NPR) mode-locked TDF laser seed (center wavelength: $1980 \mathrm{~nm}$; a repetition rate: 
30.4 MHz; a pulse width: $1.25 \mathrm{ps}$ ) followed by a TDF fiber based amplifier. The output of the laser was split into two channels by using a 10:90 coupler. The $10 \%$ port was directly monitored by a detector of a powermeter as the reference, while another $90 \%$ port was connected with the $\mathrm{Cu}_{1.8} \mathrm{Se} / \mathrm{PVA}$ composite film (saturable absorber, as same as the one used in the laser cavity). Then the output laser from the composite film was monitored by another detector of the powermeter. Note that the insertion loss introduced by two FC/PC connectors have been removed when calculating the nonlinear absorption.

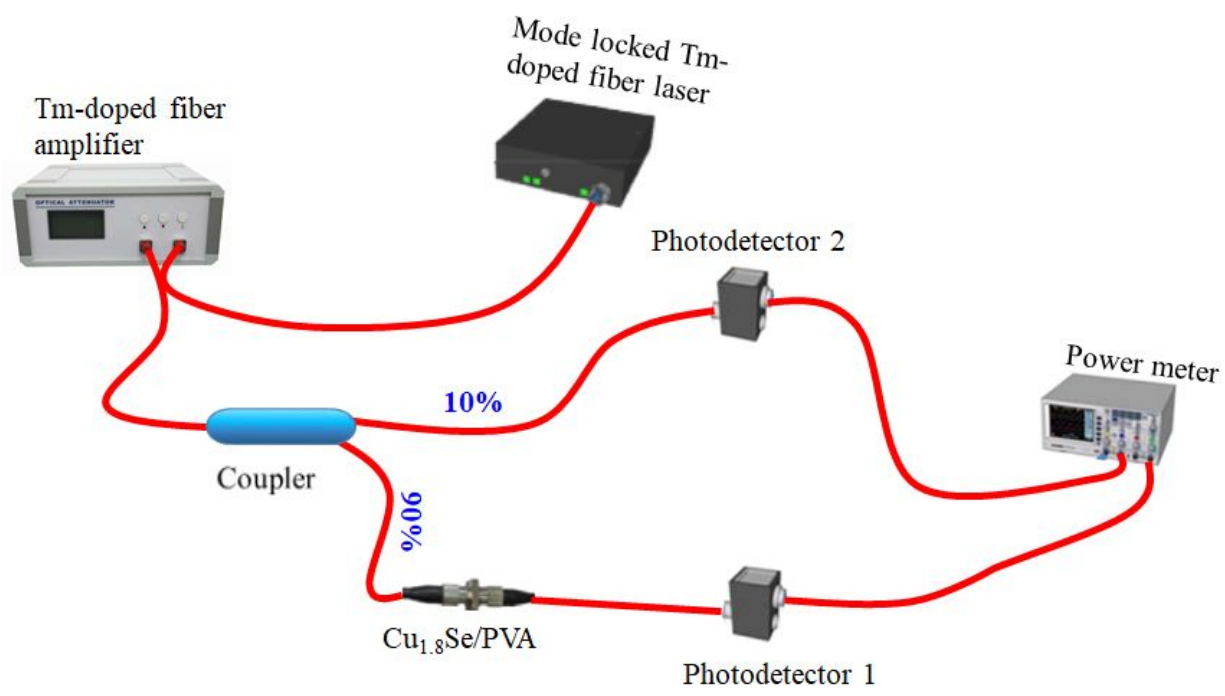

Figure S5. NLA experimental setup at $1960.3 \mathrm{~nm}$.

NLA measurement at $3000 \mathrm{~nm}$ : A typical power dependent measurement setup was built up to reveal the nonlinear absorption of $\mathrm{Cu}_{1.8} \mathrm{Se} / \mathrm{PVA}$ composite film, as shown in Figure S6. The pump source was a self-built SESAM passively mode-locked $\mathrm{Ho}^{3+} / \mathrm{Pr}^{3+}$ co-doped ZBLAN fiber laser with an output laser centered at $2868.0 \mathrm{~nm}$ (pulse duration: $20 \mathrm{ps,} \mathrm{repetition} \mathrm{rate:}$ 17.86 MHz). A beam splitter with transmittance/reflectance ration of 55:45 was employed to split the laser into two parts. The reflectance laser was focused by an uncoated $\mathrm{CaF}_{2}$ lens 
(focal length: $20 \mathrm{~mm}$, LA5315, Thorlabs) and then went through a $\mathrm{CaF}_{2}$ substrate to serve as the reference. The transmittance laser was focused by another same $\mathrm{CaF}_{2}$ lens and then went through a $\mathrm{CaF}_{2}$ substrate coated with $\mathrm{Cu}_{1.8} \mathrm{Se} \mathrm{NCs}$. Two powermeters were used to detect the output power.

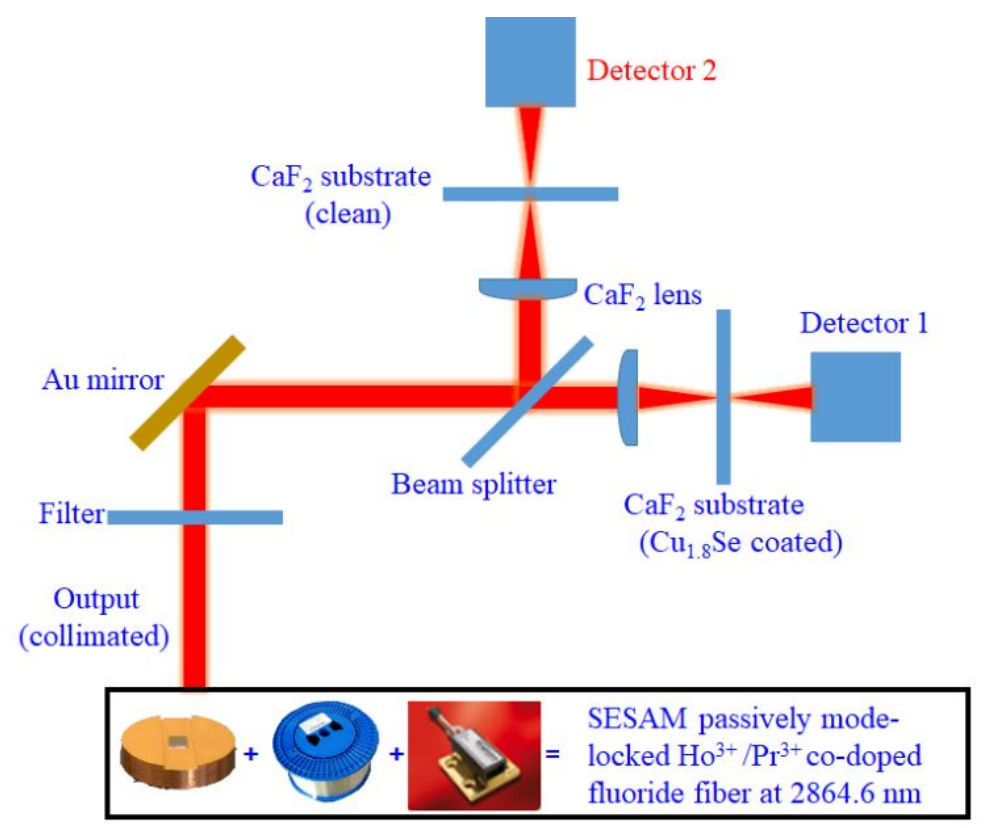

Figure S6. NLA experimental setup at $2864.6 \mathrm{~nm}$.

NLA measurements at $1064 \mathrm{~nm}$ and $1550 \mathrm{~nm}$ were operated by using Z-scan technique.

\section{CALCULATION OF NONLINEAR ABSORPTION COEFFICIENT}

According to NLO theory, the propagation can be described: ${ }^{7}$

$$
\frac{\mathrm{d} I}{\mathrm{~d} z^{\prime}}=-\alpha(I) I
$$

here, $z$ ' present the propagation length in the samples, $I$ is the incident intensity of laser. The 
total absorption $\alpha(I)$ contains two parts: a linear absorption coefficient $\alpha_{0}$ and a nonlinear absorption coefficient $\alpha_{\mathrm{NL}}$. Therefore, the total absorption can be:

$\alpha(I)=\alpha_{0}+\alpha_{\mathrm{NL}} I$

For a system, we can also describe $\alpha(I)$ using the following relationship:

$\alpha(I)=\frac{\alpha_{0}}{1+I / I_{\mathrm{s}}}+\beta I$,

where $\beta$ is nonlinear absorption coefficient. Hence, the normalized transmittance can be described by:

$$
T=\frac{1}{\sqrt{\pi}\left[\frac{\beta I_{0} L_{e f f}}{\left(1+z^{2} / z_{0}^{2}\right)}\right]} \int_{-\infty}^{+\infty} \times \ln \left[1+\frac{\beta I_{0} L_{\text {eff }}}{\left(1+z^{2} / z_{0}^{2}\right)} \exp \left(-t^{2}\right)\right] d t .
$$

Here $T, I_{0}$, and $L$ are the normalized transmittance, the peak light intensity at the focus, the thickness of the $\mathrm{Cu}_{1.8}$ Se film and the diffraction length of the beam, respectively. The effective length of the sample, $L_{\text {eff, }}$ can be defined as: $L_{\text {eff }}=\left(1-e^{-\alpha_{0} L}\right) / \alpha_{0} \cdot z_{0}$ stands for the diffraction length of the beam: $z_{0}=\pi \omega_{0}^{2} / \lambda$, with $\omega_{0}$ is the beam waist radius.

The imaginary part of the third-order nonlinear susceptibility can be expressed:

$$
\operatorname{Im} \chi^{(3)}(\mathrm{esu})=\frac{10^{-7} c^{2} n_{0}^{2}}{96 \pi^{2} \omega} \beta
$$

Where, $c, \lambda$, and $n$ are, respectively, the speed of light in the vacuum, the wavelength of incident light, and the refractive index. 
The figure of merit (FOM) is calculated as:

$$
\mathrm{FOM}=\left|\frac{\operatorname{Im} \chi^{(3)}}{\alpha_{0}}\right|
$$

\section{ADDITIONAL FIGURES}

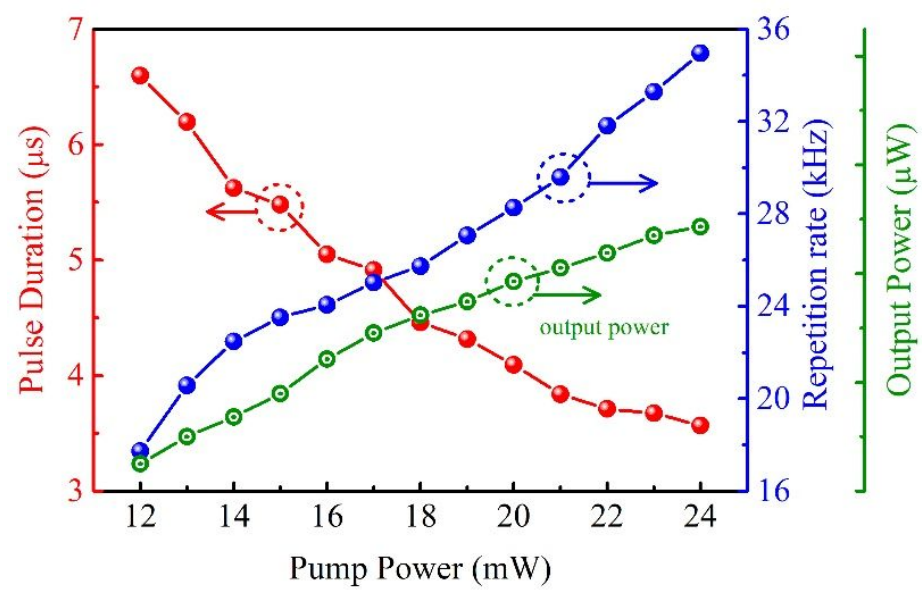

Figure S7. Q-switched characterization of EDF laser (1533.8 nm): the output power, the pulse duration and the pulse repetition rate as a function of incident pump power. 

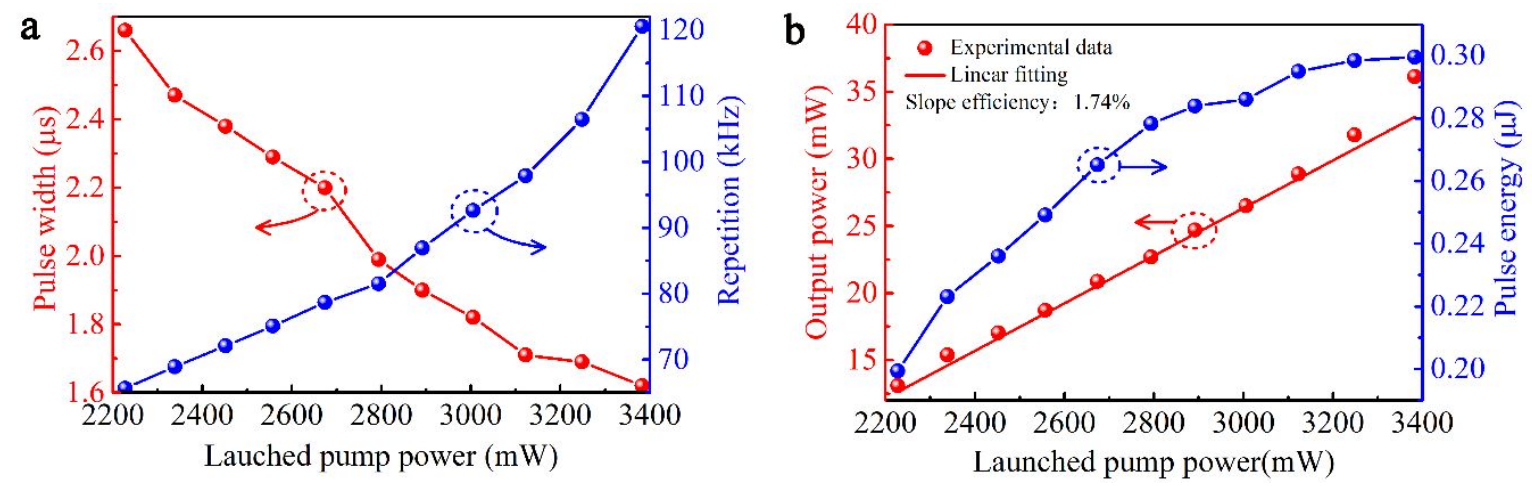

Figure S8. Q-switched characterization of TDF laser (1960.3 nm). (a) The pulse duration and the pulse repetition rate as a function of incident pump power. (b) The output power and the pulse energy as a function of incident pump power.
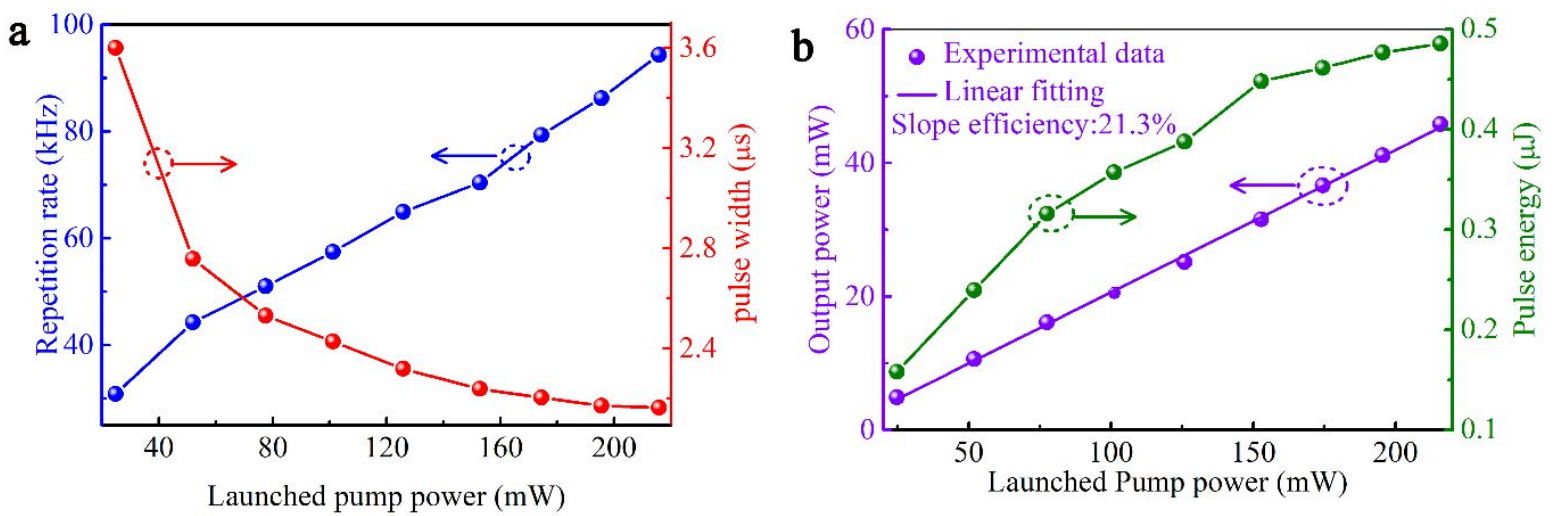

Figure S9. Q-switched characterization of Ho/Pr co-doped ZBLAN fiber laser (2864.6 nm).

(a) The pulse duration and the pulse repetition rate as a function of incident pump power. (b)

The output power and the pulse energy as a function of incident pump power. 


\section{ADDITIONAL TABLES}

Table S1 NLO parameters of several typical materials

\begin{tabular}{|c|c|c|c|c|}
\hline Laser & Sample & $\beta\left(\mathrm{cm} \mathrm{GW}^{-1}\right)$ & $\operatorname{Im} \chi^{(3)}(\mathrm{esu})$ & FOM (esu cm) \\
\hline \multirow{4}{*}{$\begin{array}{l}1030 \mathrm{~nm}, 1 \mathrm{kHz}, \\
340 \mathrm{fs}\end{array}$} & $\mathrm{MoS}_{2}{ }^{8}$ & $-(9.17 \pm 2.56) \times 10^{-2}$ & $-(6.7 \pm 1.9) \times 10^{-14}$ & $(5.7 \pm 1.6) \times 10^{-15}$ \\
\hline & $\mathrm{MoSe}_{2}{ }^{3}$ & $-(1.29 \pm 0.13) \times 10^{-2}$ & $-(9.4 \pm 1.0) \times 10^{-15}$ & $(4.22 \pm 0.27) \times 10^{-15}$ \\
\hline & $\mathrm{MoTe}_{2}{ }^{3}$ & $-(7.50 \pm 0.47) \times 10^{-3}$ & $-(5.50 \pm 0.34) \times 10^{-15}$ & $(6.38 \pm 0.39) \times 10^{-15}$ \\
\hline & Graphene $^{3}$ & $-(9.40 \pm 3.18) \times 10^{-2}$ & $-(6.9 \pm 2.3) \times 10^{-14}$ & $(4.03 \pm 1.36) \times 10^{-15}$ \\
\hline \multirow{4}{*}{$\begin{array}{l}800 \mathrm{~nm}, 1 \mathrm{kHz}, \\
100 \mathrm{fs}\end{array}$} & $\mathrm{MoS}_{2}{ }^{3}$ & $-(2.42 \pm 0.80) \times 10^{-2}$ & $-(1.38 \pm 0.45) \times 10^{-14}$ & $(1.23 \pm 0.40) \times 10^{-15}$ \\
\hline & $\mathrm{MoSe}_{2}{ }^{3}$ & $-(2.54 \pm 0.60) \times 10^{-3}$ & $-(1.45 \pm 0.34) \times 10^{-15}$ & $(6.9 \pm 1.6) \times 10^{-16}$ \\
\hline & $\mathrm{MoTe}_{2}{ }^{3}$ & $-(3.7 \pm 1.2) \times 10^{-3}$ & $-(2.13 \pm 0.66) \times 10^{-15}$ & $(1.45 \pm 0.45) \times 10^{-15}$ \\
\hline & Graphene $^{3}$ & $-(1.52 \pm 0.42) \times 10^{-2}$ & $-(8.7 \pm 2.4) \times 10^{-15}$ & $(4.9 \pm 1.4) \times 10^{-16}$ \\
\hline $1550 \mathrm{~nm}, 35 \mathrm{fs}$ & Black Phosphor-us ${ }^{9}$ & $-0.15 \times 10^{-3}$ & N/A & N/A \\
\hline $\begin{array}{l}800 \mathrm{~nm}, \\
1 \mathrm{kHz}, 100 \mathrm{fs}\end{array}$ & Black Phosphor-us ${ }^{10}$ & $-(4.08 \pm 0.11) \times 10^{-3}$ & N/A & N/A \\
\hline $1550 \mathrm{~nm}, 150 \mathrm{fs}$ & SWNTs ${ }^{11}$ & N/A & $10^{-10}$ & N/A \\
\hline $800 \mathrm{~nm}, 220 \mathrm{fs}$ & Au nanorodes 12 & -1.5 & $-1.2 \times 10^{-12}$ & $3 \times 10^{-14}$ \\
\hline $1250 \mathrm{~nm}, 150 \mathrm{fs}$ & ITO $^{13}$ & $-8 \times 10^{3}$ & N/A & $\mathrm{N} / \mathrm{A}$ \\
\hline $1260 \mathrm{~nm}$ & $\mathrm{ITO} / \mathrm{Au}$ antenna ${ }^{14}$ & $2.4 \times 10^{5}$ & N/A & N/A \\
\hline $800 \mathrm{~nm}, 120 \mathrm{fs}$ & $\mathrm{Cu}_{1.8} \mathrm{Se} \mathrm{NCs}{ }^{[a]}$ & -97.5 & $-5.9 \times 10^{-11}$ & $7.2 \times 10^{-14}$ \\
\hline $1064 \mathrm{~nm}, 120 \mathrm{fs}$ & $\mathrm{Cu}_{1.8} \mathrm{Se} \mathrm{NCs}^{[\mathrm{a}]}$ & -210 & $-1.7 \times 10^{-10}$ & $2.1 \times 10^{-14}$ \\
\hline $1550 \mathrm{~nm} 120 \mathrm{fs}$ & $\mathrm{Cu}_{1.8} \mathrm{Se} \mathrm{NCs}^{[\mathrm{a}]}$ & $-1.4 \times 10^{3}$ & $-1.65 \times 10^{-10}$ & $2.1 \times 10^{-14}$ \\
\hline
\end{tabular}

aThis work

\section{REFERENCE:}

1. Luther, J. M.; Jain, P. K.; Ewers, T.; Alivisatos, A. P., Localized Surface Plasmon Resonances Arising from Free Carriers in Doped Quantum Dots. Nat. Mater. 2011, 10, 361.

2. Mansour, B. A.; Demian, S. E.; Zayed, H. A., Determination of the Effective Mass for Highly Degenerate Copper Selenide from Reflectivity Measurements. J. Mater. Sci.-Mater. El. 1992, 3, 249-252.

3. Butler, M. A., Photoelectrolysis and Physical Properties of the Semiconducting Electrode $\mathrm{WO}_{2}$ J. Appl. Phys. 1977, 48, 1914-1920.

4. Wilson, B. C.; Jacques, S. L., Optical Reflectance and Transmittance of Tissues: Principles 
and Applications. IEEE J. Quantum Elect. 1990, 26, 2186-2199.

5. Tian, X.; Wei, R.; Liu, M.; Zhu, C.; Luo, Z.; Wang, F.; Qiu, J., Ultrafast Saturable Absorption in $\mathrm{TiS}_{2}$ Induced by Non-Equilibrium Electrons and the Generation of $\mathrm{A}$ Femtosecond Mode-Locked Laser. Nanoscale 2018, 10, 9608-9615.

6. Tian, X.; Luo, H.; Wei, R.; Zhu, C.; Guo, Q.; Yang, D.; Wang, F.; Li, J.; Qiu, J., An Ultrabroadband Mid-Infrared Pulsed Optical Switch Employing Solution-Processed Bismuth Oxyselenide. Adv. Mater. 2018, 30, 1801021.

7. Hasan, T.; Sun, Z.; Wang, F.; Bonaccorso, F.; Tan, P. H.; Rozhin, A. G.; Ferrari, A. C., Nanotube-Polymer Composites for Ultrafast Photonics. Adv. Mater. 2009, 21, 3874-3899.

8. Wang, K.; Feng, Y.; Chang, C.; Zhan, J.; Wang, C.; Zhao, Q.; Coleman, J. N.; Zhang, L.; Blau, W. J.; Wang, J., Broadband Ultrafast Nonlinear Absorption and Nonlinear Refraction of Layered Molybdenum Dichalcogenide Semiconductors. Nanoscale 2014, 6, 10530-10535.

9. Wang, Y.; Huang, G.; Mu, H.; Lin, S.; Chen, J.; Xiao, S.; Bao, Q.; He, J., Ultrafast Recovery Time and Broadband Saturable Absorption Properties of Black Phosphorus Suspension. Appl.Phys. Lett. 2015, 107, 091905.

10. Lu, S. B.; Miao, L. L.; Guo, Z. N.; Qi, X.; Zhao, C. J.; Zhang, H.; Wen, S. C.; Tang, D. Y.; Fan, D. Y., Broadband Nonlinear Optical Response in Multi-Layer Black Phosphorus: An Emerging Infrared and Mid-Infrared Optical Material. Opt. Express 2015, 23, 11183-11194.

11. Chen, Y.-C.; Raravikar, N. R.; Schadler, L. S.; Ajayan, P. M.; Zhao, Y.-P.; Lu, T.-M.; Wang, G.-C.; Zhang, X.-C., Ultrafast Optical Switching Properties of Single-Wall Carbon Nanotube Polymer Composites at $1.55 \mu \mathrm{m}$. Appl. Phys. Lett. 2002, 81, 975-977.

12. Elim, H. I.; Yang, J.; Lee, J.-Y.; Mi, J.; Ji, W., Observation of Saturable and Reverse- 
Saturable Absorption at Longitudinal Surface Plasmon Resonance in Gold Nanorods. Appl. Phys. Lett. 2006, 88, 083107.

13. Alam, M. Z.; De Leon, I.; Boyd, R. W., Large Optical Nonlinearity of Indium Tin Oxide in Its Epsilon-near-Zero Region. Science 2016, 352, 795-797.

14. Alam, M. Z.; Schulz, S. A.; Upham, J.; De Leon, I.; Boyd, R. W., Large Optical Nonlinearity of Nanoantennas Coupled to An Epsilon-Near-Zero Material. Nat. Photonics 2018, $12,79-83$. 